\title{
Title: Treatment and long-term outcome in primary nephrogenic diabetes insipidus
}

Authors: Sergio C Lopez-Garcia ${ }^{1,2}$, Mallory L Downie ${ }^{1,2}$, Ji Soo Kim², Olivia Boyer ${ }^{3}$, Stephen B Walsh ${ }^{1}$, Tom Nijenhuis ${ }^{4}$, Svetlana Papizh ${ }^{5}$, Pallavi Yadav ${ }^{6}$, Ben C Reynolds ${ }^{7}$, Stéphane Decramer ${ }^{8}$, Martine Besouw ${ }^{9}$, Manel Perelló Carrascosa ${ }^{10}$, Claudio La Scola ${ }^{11}$, Francesco Trepiccione ${ }^{12}$, Gema Ariceta ${ }^{13}$, Aurélie Hummel ${ }^{14}$, Claire Dossier ${ }^{15}$, John A Sayer ${ }^{16,17,18}$, Martin Konrad ${ }^{19}$, Mandy G Keijzer-Veen ${ }^{20}$, Atif Awan $^{21}$, Biswanath Basu ${ }^{22}$, Dominique Chauveau ${ }^{23}$, Leire Madariaga ${ }^{24}$, Linda Koster-Kamphuis ${ }^{25}$, Mónica Furlano ${ }^{26}$, Miriam Zacchia ${ }^{12}$, Pierluigi Marzuillo ${ }^{27}$, Yincent $\mathrm{Ts}^{28}$, Ismail Dursun ${ }^{29}$, Ayse Seda Pinarbasi $^{29}$, Despoina Tramma ${ }^{30}$, Ewout J. Hoorn ${ }^{31}$, Ibrahim Gokce ${ }^{32}$, Kathleen Nicholls ${ }^{33,34}$, Loai A. Eid $^{35}$, Lisa Sartz ${ }^{36}$, Michael Riordan ${ }^{21}$, Nakysa Hooman ${ }^{37}$, Nikoleta Printza ${ }^{38}$, Olivier Bonny ${ }^{39}$, Pedro Arango Sancho ${ }^{40}$, Raphael Schild ${ }^{41}$, Rajiv Sinha ${ }^{42}$, Stefano Guarino ${ }^{27}$, Victor Martinez Jimenez ${ }^{43}$, Lidia Rodríguez Peña ${ }^{44}$, Hendrica Belge ${ }^{45}$, Olivier Devuyst ${ }^{46}$, Tanja Wlodkowski ${ }^{47}$, Francesco Emma ${ }^{48}$, Elena Levtchenko ${ }^{49}$, Nine V.A.M. Knoers ${ }^{50,51}$, Daniel G. Bichet ${ }^{52}$, Franz Schaefer ${ }^{47}$, the European NDI Consortium*, Robert Kleta ${ }^{1,2}$ and Detlef Bockenhauer ${ }^{1,2}$.

${ }^{1}$ UCL Department of Renal Medicine, London, UK; ${ }^{2}$ Paediatric Nephrology Unit, Great Ormond Street Hospital, London, UK; ${ }^{3}$ Department of Pediatric Nephrology, Reference center for Hereditary Kidney Diseases (MARHEA), Laboratory of Hereditary Kidney Diseases, Imagine Institute, INSERM U1163, Paris Descartes University, Necker Hospital, APHP, Paris, France; ${ }^{4}$ Department of Nephrology, Radboud Institute for Molecular Life Sciences, Radboudumc Expertisecentrum Zeldzame Nierziekten, Radboud University Medical Center, Nijmegen, the Netherlands; ${ }^{5}$ Department of hereditary and acquired kidney diseases, Research and Clinical Institute for Pediatrics at the Pirogov Russian National Research Medical University, Moscow, Russia; ${ }^{6}$ Department of Paediatric Nephrology, Leeds Teaching Hospitals NHS Trust, Leeds, UK; 'Department of Paediatric Nephrology, Royal Hospital for Children, Glasgow, G51 4TF, Scotland; ${ }^{8}$ Centre Hospitalier Universitaire de Toulouse, Service de Nephrologie Pediatrique, Hopital des Enfants, Centre De Reference des Maladies Rénales Rares du Sud Ouest, Toulouse, France; ${ }^{9}$ Department of Paediatric Nephrology, University of Groningen, University Medical Center Groningen, Groningen, The Netherlands; ${ }^{10}$ Department of Nephrology, Vall d'Hebron University Hospital, Barcelona, Spain; ${ }^{11}$ Nephrology and Dialysis Unit, Department of Woman, Child and Urological Diseases, Azienda Ospedaliero-Universitaria Sant'Orsola-Malpighi, Bologna, Italy; ${ }^{12}$ Department of Translational Medical Sciences, University of Campania "L.Vanvitelli", Naples, Italy; ${ }^{13}$ Department of Paediatric Nephrology, Hospital Universitario Vall d'Hebron, Barcelona, Spain; ${ }^{14}$ Department of Nephrology and Transplantation, Centre de Référence des Maladies Rénales Héréditaires de I'Enfant et de I'Adulte, Necker Hospital, APHP, Paris, France; ${ }^{15}$ Department of Paediatric Nephrology, Hôpital Robert-Debre, Paris, France; ${ }^{16}$ Translational and Clinical Medicine Institute, Faculty of Medical Sciences, Newcastle University, Central Parkway, Newcastle Upon Tyne, NE1 3BZ UK; ${ }^{17}$ Renal Services, The Newcastle upon Tyne Hospitals NHS Foundation Trust, Newcastle upon Tyne, NE7 7DN, UK; ${ }^{18} \mathrm{NIHR}$ Newcastle Biomedical Research Centre, Newcastle University, Newcastle upon Tyne, NE4 5PL, UK; ${ }^{19}$ University Children's Hospital, Münster, Germany; ${ }^{20}$ Department of pediatric nephrology, Wilhelmina Children's Hospital, University Medical Center Utrecht, Utrecht, The Netherlands; ${ }^{21}$ Department of Paediatric Nephrology, Temple Street, Dublin, Ireland; ${ }^{22}$ Division of Pediatric Nephrology, Department of Pediatrics, N. R. S. Medical College \& Hospital, Kolkata, India; ${ }^{23}$ Department of Nephrology and Transplantation, Centre de Référence des Maladies Rénales Rares, Centre Hospitalier Universitaire de Toulouse, Université Toulouse-III, France; ${ }^{24}$ Department of Paediatric Nephrology, Cruces University Hospital, IIS Biocruces-Bizkaia, University of the Basque Country, Bizkaia, Spain; ${ }^{25}$ Department of Paediatric Nephrology, Amalia Children's Hospital, Radboud University Medical Center, Nijmegen, The Netherlands; ${ }^{26}$ Inherited Kidney Diseases, Nephrology 
Department, Fundació Puigvert, Instituto de Investigaciones Biomédicas Sant Pau (IIB-Sant Pau), Department of Medicine-Universitat Autónoma de Barcelona, REDinREN, Instituto de Investigación Carlos III, Barcelona, Spain; ${ }^{27}$ Department of Women, Child and of General and Specialized Surgery, Università degli Studi della Campania "Luigi Vanvitelli", Naples, Italy; ${ }^{28}$ Department of Paediatric Nephrology, Great North Children's Hospital, Newcastle Upon Tyne, UK; ${ }^{29}$ Erciyes University Faculty of Medicine, Department of Paediatrics, Division of Nephrology, Kayseri, Turkey; ${ }^{30} 4$ th Department of Pediatrics, School of Medicine, Faculty of Health Sciences, Aristotle University of Thessaloniki, Papageorgiou General Hospital Thessaloniki, Greece; ${ }^{31}$ Department of Internal Medicine, Division of Nephrology and Transplantation. Erasmus Medical Center, University Medical Center Rotterdam, Rotterdam, The Netherlands; ${ }^{32}$ Department of Paediatric Nephrology, Marmara University, Faculty of Medicine, İstanbul, Turkey; ${ }^{33}$ Department of Nephrology, Royal Melbourne Hospital Parkville, Australia; ${ }^{34}$ University of Melbourne, Parkville, Australia; ${ }^{35}$ Department of Paediatric Nephrology, Dubai Hospital - Dubai Health Authority, UAE; ${ }^{36}$ Department of Clinical Sciences, Paediatric nephrology, Skåne University hospital, Lund University, Lund, Sweden; ${ }^{37}$ Aliasghar Clinical Research Development Center(ACRDC), Aliasghar Children Hospital, Iran University of Medical Sciences Tehran, Iran; ${ }^{38} 1$ st Paediatric Department, Aristotle University, Thessaloniki, Greece; ${ }^{39}$ Service of Nephrology, Rue du Bugnon 17, Lausanne University Hospital, 1011 Lausanne, Switzerland; ${ }^{40}$ Department of Paediatric Nephrology and Transplantation, Hospital Sant Joan De Déu, Esplugues De Llobregat, Barcelona, Spain; ${ }^{41}$ Department of Paediatrics, University Medical Center Hamburg- Eppendorf, Germany; ${ }^{42}$ Division of Paediatric Nephrology, Institute of Child Health, Kolkata, India; ${ }^{43}$ Department of Nephrology, Hospital Clínico Universitario Virgen de la Arrixaca, Murcia, Spain; ${ }^{44}$ Section of Medical Genetics, Department of Pediatrics, Hospital Clínico Universitario Virgen de la Arrixaca, Murcia, Spain; ${ }^{45}$ Center of Human Genetics, Institut de Pathologie et Genetique, Gosselies, Belgium; ${ }^{46}$ Institute of Physiology, Zurich Center for Integrative Human Physiology (ZHIP), Mechanism of Inherited Kidney Disorders Group, University of Zurich, Switzerland; ${ }^{47}$ Department of Paediatric Nephrology, University Children's Hospital, Heidelberg, Germany; ${ }^{48}$ Division of Nephrology, Department of Pediatric Subspecialties, Bambino Gesù Children's Hospital, IRCCS, Rome, Italy; ${ }^{49}$ University Hospital Leuven, Belgium; ${ }^{50}$ Department of Genetics, Center for Molecular Medicine, University Medical Centre Utrecht, Utrecht University, Utrecht, The Netherlands, ${ }^{51}$ Department of Genetics, University of Groningen, University Medical Centre Groningen, Groningen, The Netherlands; ${ }^{52}$ Nephrology Service, Departments of Medicine, Pharmacology and Physiology, University of Montreal, Montreal, Quebec, Canada.

\section{*The European NDI consortium consists of the authors, as well as:}

Anna Wasilewska ${ }^{53}$, Germana Longo ${ }^{54}$, Laura Espinosa ${ }^{55}$, Marius Miglinas ${ }^{56}$, Ramona Stroescu ${ }^{57,58}$, Shafa Huseynova ${ }^{59}$, Stella Stabouli ${ }^{38}$, Vijaya Sathyanarayana ${ }^{28}$, Andreea G Andronesi ${ }^{60,61}$, Deirdre Hahn $^{62}$, Deepak Sharma ${ }^{63}$, Edita Petrosyan ${ }^{64}$, Eleni Frangou ${ }^{65}$, Nilufar Mohebbi ${ }^{66}$, Nida Temizkan Dinçel ${ }^{67}$, Philippe Braconnier ${ }^{39}$, Rodney D Gilbert ${ }^{68}$, Adamu Sambo ${ }^{69}$, Velibor Tasic $^{70}$ and Thomas Henne $^{41}$.

${ }^{53}$ Department of Paediatric Nephrology, Medical University of Bialystok, Poland; ${ }^{54}$ Pediatric Nephrology, Dialysis and Transplant Unit, Department of Woman's and Child's Health, Azienda Ospedaliera di Padova - University of Padova; ${ }^{55}$ Department of Paediatric Nephrology. Hospital Universitario La Paz, Madrid, Spain; ${ }^{56}$ Vilnius university, Nephrology Centre, Santaros klinikos, Vilnius, Lithuania; ${ }^{57}$ Louis Turcanu Emergency Hospital for Children, Timisoara, Romania; ${ }^{58}$ Victor Babes University of Medicine and Pharmacy, Timisoara, Romania; ${ }^{59}$ Department of Paediatric Nephrology, Karadeniz Technical University, Turkey; ${ }^{60}$ Department of Nephrology, Carol Davila University of 
Medicine and Pharmacy, Bucharest, Romania; ${ }^{61}$ Nephrology Department, Fundeni Clinical Institute, Bucharest, Romania; ${ }^{62}$ Children's Hospital Westmead, Sydney, Australia; ${ }^{63}$ Nephrology, Dialysis \& Kidney Transplantation. CMD \& Founder Ketav Kalp Healthcare \& Research Private Limited. New Delhi. India; ${ }^{64}$ Department of Nephrology, Russian State Medical University, Moscow, Russia; ${ }^{65}$ Department of Nephrology, Limassol General Hospital, Limassol, Cyprus; ${ }^{66}$ Division of Nephrology, University Hospital Zurich, Zurich, Switzerland; ${ }^{67}$ Department of Paediatric Nephrology, Dr Behcet Uz Children's Hospital, University of Health Sciences, Izmir, Turkey; ${ }^{68}$ Wessex Regional Paediatric Nephro-urology Service, Southampton Children's Hospital, Faculty of Medicine, University of Southampton, UK; and ${ }^{69}$ Department of Paediatrics, Gloucester Hospital, UK; and ${ }^{70}$ University Children's Hospital, Medical School, Skopje, Macedonia.

\section{Corresponding author:}

Prof. Dr. D. Bockenhauer

Department of Pediatric Nephrology

Great Ormond Street Hospital NHS Trust

Great Ormond Street

London WC1N 3JH

Tel: +442074059200

d.bockenhauer@ucl.ac.uk

key words: nephrogenic diabetes insipidus, chronic kidney disease, flow uropathy, AVPR2 and AQP2

word count: abstract: 248

main text: 3442 


\section{Abstract}

BACKGROUND: Primary nephrogenic diabetes insipidus (NDI) is a rare disorder and little is known on treatment practices and long-term outcome.

METHODS: Paediatric and adult nephrologists contacted through European professional organizations entered data in an online form.

RESULTS: Data was collected on 315 patients (22 countries, male $84 \%$, adults $35 \%$ ). Mutation testing had been performed in 270 (86\%); pathogenic variants were identified in 258 (96\%). The median (range) age at diagnosis was $0.6(0.0-60)$ years and at last follow-up 14.0 (0.1-70) years. In adults, height was normal with a mean standard deviation score (SDS) of $-0.39( \pm 1.0)$, yet there was increased prevalence of obesity (body mass index $\left.>30 \mathrm{~kg} / \mathrm{m}^{2}\right)(41 \%$ versus $16 \%$ European average; $\mathrm{p}<0.001)$. There was also increased prevalence of chronic kidney disease (CKD) stage $\geq 2$ in children (32\%) and adults (48\%). Evidence of flow uropathy was present in $38 \%$. A higher proportion of children than adults $(85 \%$ versus $54 \% ; p<0.001)$ received medications to reduce urine output.

Patients 25 years or older were less likely to have a university degree than the European average ( $21 \%$ versus $35 \%, p=0.003$ ) but full-time employment was similar. Mental health problems, predominantly attention-deficit hyperactivity disorder (16\%) were reported in $36 \%$ of patients.

CONCLUSION: This large NDI cohort shows an overall favourable outcome with normal adult height and only mild to moderate CKD in most. Yet, while full time employment was similar to the European average, educational achievement was lower, and more than half had urological and/or mental health problems. 
- What is already known about this subject:

- Primary nephrogenic diabetes insipidus is a rare inherited disorder of impaired urinary concentration

- Previous literature predominantly concerns case reports, paediatric single-centre cohorts or genetic aspects

- Data are lacking on long-term outcome

\section{- What this study adds:}

- Largest cohort so far reported and inclusion of 110 adults up the age of 70 years provides important data on long-term outcome

- Final height is essentially normal and chronic kidney disease is mild in most

- There is a high prevalence of obesity, flow uropathy and mental health problems

\section{- What impact this may have on practice or policy:}

- This study provides unprecedented information on long-term outcome that directly informs the management and prognosis of patients with NDI 


\section{Introduction}

Primary nephrogenic diabetes insipidus (NDI) is a rare inherited condition of impaired urinary concentration [1]. Patients typically present in the first year of life with failure-to-thrive and vomiting. An affected adult will typically void around 10-12 litres of urine in 24 hours. Reported complications include impaired school performance and behavioural abnormalities, such as attention deficit hyperactivity disorder (ADHD) [2]. Patients may also develop a flow uropathy, dilatation of the urinary tract, because of the large urine volumes. Two genes have been identified as causative for NDI: AVPR2, encoding the type 2 vasopressin receptor in the kidney and $A Q P 2$, encoding the water channel expressed on the apical side of the principal cell in the collecting duct [1]. AVPR2 is located on the Xchromosome and mutations are inherited in an X-linked recessive manner, whereas AQP2 mutations are typically inherited in an autosomal recessive pattern, with rare autosomal dominant cases reported [2]. Mutations in AVPR2 are roughly 10 times more frequent than AQP2. Consequently, the vast majority of patients with NDI are male. Primary NDI is rare with an estimated incidence of approximately 1 in 100,000 and the few data available on long-term outcome are mostly based on small single-centre cohorts. We performed a cross-sectional cohort study to gather information on kidney function, flow uropathy, auxology, mental health, education, employment and living arrangements in patients with primary NDI across all ages. 


\section{PATIENTS AND METHODS}

\section{Clinical data}

An email was sent to the membership of the European Reference Network for Rare Kidney Diseases (ERKNet), the European Renal Association - European Dialysis and Transplant Association (ERA-EDTA) and the European Society for Paediatric Nephrology (ESPN) through their respective working groups for inherited kidney diseases, inviting clinicians to provide data on patients with a clinical diagnosis of inherited NDI. The email contained a link to an online data form which was open from the $26^{\text {th }}$ of June to the $31^{\text {st }}$ of August 2019.

A total of 27 questions were asked about demographics, auxology, treatment, kidney function, comorbidities, such as hydronephrosis, bladder dysfunction and mental health problems. A list of all questions is provided in supplementary table 1.

In cases of missing information or if provided data points were noted to be outliers, corresponding clinicians were contacted via email for completion and/or verification of data. Data were deemed adequate for analysis if $<5$ items were missing, and the information provided was confirmed by the responsible clinician (supplementary table 2).

We used the age of 18 years to separate the cohort into a paediatric and adult group. The only exception is estimated glomerular filtration rate (eGFR), as the "Schwartz" formula is recommended up to the age of 20 years [3].

\section{Genotype-phenotype analysis}

For genotype-phenotype analysis, we divided the cohort according to the genetic information into the following 4 groups: 1. Negative: no causative mutations identified in AVPR2 and AQP2; 2. Untested: genetic testing not performed; or causative mutation(s) had been identified in either 3. AVPR2 or 4. $A Q P 2$. We also separated between missense and predicted loss of function (pLoF) mutations. 


\section{Auxology}

Height data were normalised and expressed as SDS (Standard Deviation Score). For children, calculations were done according to WHO data [4, 5], while for adults the US 2000 CDC growth charts (according to National Health and Nutrition Survey (NHANES) data) were used [6]. Normal height was defined as a calculated SDS $\geq-2.00$.

Paediatric weight data were normalised and expressed as SDS (Standard Deviation Score), also using the WHO data. The body mass index (BMI) was calculated and defined as underweight $(<18.5)$, normal (18.5-24.9), overweight (25.0-29.9) or obese $\left(\geq 30.0 \mathrm{~kg} / \mathrm{m}^{2}\right)$, according to standard convention [7].

\section{Kidney function}

The eGFR in adults (>20 years old) was calculated using the modification of diet in renal disease (MDRD) formula $[3,8]$. For children (2-20 years), we used the modified "Schwartz" formula [3]. Prevalence of CKD was calculated and expressed in stages 1-5 according to KDIGO guidelines [9]. For comparison with NHANES III cohort [10], data from patients aged 20-60 years ( $\mathrm{N}=76)$ was used for subsequent analysis of CKD prevalence. As there were only 7 patients $>60$ years, meaningful comparison for that age group was not possible.

\section{Psychosocial information}

We used a published estimate of a $5 \%$ worldwide prevalence of ADHD, while European epidemiological data on educational attainment and employment were extracted from the EuroStat database [11-13]. Mental Health disorders were classified according to DSM-5 criteria [14].

\section{Gross Domestic Product (GDP) per capita (\$)}

GDP per capita was based data on from The World Bank [15]. For analysis, we defined a low and high subgroup based on a $15.000 \$ /$ year cut-off, as it roughly divided the cohort in half. 


\section{Statistics}

Analysis was done in IBM SPSS Statistics for Windows version 24.0 (Armonk, NY: IBM Corp.).

Kolmogorov-Smirnov test was performed to assess normality of the data. Data following a normal distribution were expressed as mean \pm standard deviation (SD). Non-normally distributed data were expressed as median with either range or interquartile range (IQR). Statistical significance for categorical/dichotomous variables was performed with the Pearson Chi-square test. The Student-T test was used to compare the means between two groups of parametric data and one-way ANOVA test for three or more different groups. Mann-Whitney U-test was used to compared mean ranks between dichotomous variables. 


\section{RESULTS}

Demographic and genetic data

A total of 315 cases (from 22 countries, supplementary table 3) were available for final analysis. Gender distribution was as expected unequal with 266 (84\%) males and 49 (16\%) females (Figure 1a). Genetic analysis in the two known NDI genes had been performed in 270 cases (86\%) and of these 258 (96\%) were found to have causative mutations ( $A V P R 2=216$ and $A Q P 2=42) ; 45$ individuals had not been genetically tested (Figure 1b). A list of all reported mutations is provided in supplementary table

4. Distribution of the reported ethnicities showed a high proportion of white/European (81\%), followed by Asian (10\%) (Figure 1c).

The median age (range) was $14.0(0.1-70)$ years at last follow up (Figure $1 \mathrm{~d}$ ) and 110 patients (35\%) were adults ( $\geq 18$ years old).

Age at diagnosis

The majority $(58 \%, \mathrm{~N}=179)$ of patients were diagnosed in the first year of life, yet $6 \%$ were diagnosed during adulthood (of these, $79 \%$ had pathogenic mutations). The median age (IQR) at diagnosis was $0.6(0.1-2.0)$ years with no significant difference between the genetic groups (Figure 2a). Analysis by mutation type (Figure $\mathbf{2} \mathbf{b}$ ) showed an earlier diagnosis in the group with predicted loss of function (pLoF) compared to missense mutations $(0.3[0.0-1.0]$ versus $0.7[0.1-2$.$] years; p=0.037)$. Male patients with pathogenic mutations in AVPR2 were significantly younger at diagnosis than females (0.5 [0.1-1.5] versus 3.1 [1.0-18.3] years; $p=0.01$ ) (Figure $2 \mathrm{c}$ ). Moreover, patients living in countries with higher GDP per capita had an earlier age at diagnosis compared with those in low-income countries (0.5 [0.0-1.4] versus $0.7[0.3-3.0]$ years; $p=0.011)$ (Figure $2 d$ ).

\section{Auxology}


Reported weight and height SDS for both paediatric and adult patients were analysed for the 4 genetic groups (Table 1). There was no significant difference for weight SDS between the paediatric groups (Figure 3a). In contrast, there was a significant difference $(p=0.03)$ in adults, showing an increased BMI (Mean \pm SD) in patients with confirmed mutations $(29.3 \pm 6.2)$ compared to those with undefined genetic diagnosis (26.0 \pm 5.5$)$. Secondary analysis for adult patients from EU28 countries $(\mathrm{N}=68)$ showed a significantly elevated proportion of obese individuals in the NDI cohort compared to the reference population (41\% versus $16 \% ; p<0.001$ ) (Figure $3 \mathbf{b}$ ). This scenario was reversed for height: paediatric patients with confirmed mutations had a significantly $(p<0.05)$ lower height SDS compared to those in the negative group (Figure 3c). This difference was not seen in the adult patients (Figure 3d). Overall $12.6 \%$ of individuals from the entire cohort did have a low height (SDS<-2.0) at last follow up.

\section{Kidney function}

The prevalence of CKD stage $\geq 2$ in the paediatric age ( $2-20$ years old) was $32 \%$, with most $(85 \%)$ in stage 2 (Figure 4a).

Mean $( \pm S D)$ eGFR at last follow up in adults was $87( \pm 36) \mathrm{ml} / \mathrm{min} / 1.73 \mathrm{~m}^{2}$ and was broadly similar across the genetic groups: Negative $74( \pm 35)$, Untested $84( \pm 44), A V P R 287( \pm 32)$ and AQP2 $97( \pm 43)$ $\mathrm{ml} / \mathrm{min} / 1.73 \mathrm{~m}^{2} ;(\mathrm{p}=0.8)$. Of the 87 adult patients ( $\geq 20$ years) with eGFR data available, $42(48 \%)$ had CKD stage $\geq 2$ and one patient with end-stage kidney disease (ESKD) was noted (Figure 4b). The linear estimation of kidney function decline in adults showed a loss of $1.4 \mathrm{ml} / \mathrm{min} / 1.73 \mathrm{~m}^{2} /$ year from 20 years of age with a starting eGFR of $110 \mathrm{ml} / \mathrm{min} / 1.73 \mathrm{~m}^{2}$ (Figure $\left.4 \mathrm{c}\right)$. CKD stage $\geq 2$ was significantly $(p<0.001$ ) more common (44\%) in NDI patients age $20-60$ years compared to the NHANES III population (26\%). The difference was especially pronounced at younger adult age (20-39 years) with a prevalence of $32 \%$ ( $\mathrm{N}=57)$, compared to $14 \%$ in NHANES III (Figure 4d). In adult patients, no significant association was found between kidney function and genetic group or GDP. However, in the paediatric group (2-20 
years), mean $( \pm S D)$ eGFR was higher in countries with high income compared to low income $(110( \pm 29)$ versus $\left.95( \pm 29) \mathrm{ml} / \mathrm{min} / 1.73 \mathrm{~m}^{2} ; \mathrm{p}=0.001\right)$ (Figures $4 \mathrm{e}, \mathrm{f}$ ).

\section{Treatment}

From the entire cohort ( $\mathrm{N}=315), 67 \%$ were treated (at last follow-up) with thiazide diuretics, $35 \%$ with potassium-sparing diuretics and $31 \%$ with nonsteroidal anti-inflammatory drugs (NSAIDs) (supplementary table 6). The age distribution for thiazide and NSAID treatments are shown in Figure $\mathbf{5 a}$ and Figure 5b. Prescription of these drugs was significantly $(p<0.001)$ more prevalent in patients under 20 years of age: $44 \%$ of adult patients were not prescribed either of these drugs in contrast to 15\% of children (Figure 5c). With regards to tube feeding for long-term enteral feeding/hydration, $18 \%(\mathrm{~N}=59)$ had had a nasogastric tube and $7 \%(\mathrm{~N}=23)$ a gastrostomy in place at some point $(\mathrm{N}=247)$. The median (IQR) age for tube insertion was $<1$-month $(<1$ to10) and for removal 2.0 (1.0-3.8) years.

\section{Complications}

In total $45 \%$ of patients had evidence of flow uropathy. The prevalence of hydronephrosis, bladder dysfunction or both ( $\mathrm{N}=266$ ) were $34 \%, 23 \%$ and $12 \%$, respectively (Figure 6a). Of note eGFR was significantly $(p=0.001)$ lower in those with urological complications compared to those without (90 versus $103 \mathrm{ml} / \mathrm{min} / 1.73 \mathrm{~m}^{2}$ ) (Figure $6 \mathbf{b}$ ). Patients of Asian ethnicity had a higher presence of flow uropathy $(20 / 27 ; 74 \%)$ than Europeans $(83 / 207 ; 40 \%)(p<0.001)$. Other factors like gender, genetics or medication prescription were not significantly associated with an increased risk of flow uropathy. Primary nocturnal enuresis was reported in $38 \%$ of patients $\geq 6$ years old $(N=250)$ and the median (IQR) age at achieving nocturnal continence was 8 (6-12) years (Figure 6c). There was no significant correlation between enuresis and flow uropathy.

\section{Mental health}


Mental health problems were reported in $36 \%$ of the entire cohort $(\mathrm{N}=178)$, and in $41 \%$ of adults, which is significantly $(p<0.001)$ higher than in the general European population (25\%) [16]. A diagnosis of ADHD was reported in $16 \%$, which again was significantly $(p<0.001)$ higher than in the general population (5\%) (Figure 7a). Among demographic variables that correlated positively $(p<0.05)$ with ADHD were: male gender, low GDP and European ethnicity; in contrast, there was no significant correlation with underlying gene, type of mutation or medication prescription.

The second most frequent mental health diagnosis was intellectual disability, reported in $9 \%(\mathrm{~N}=16)$. There was no significant association with gender, ethnicity, age at diagnosis and GDP. Supplementary table 7 details the different mental health disorders identified in our cohort.

\section{Education, employment and living arrangements}

For those individuals $(\mathrm{N}=41)$ within the $25-54$ years age range and belonging to one of the EU-28 countries, the highest level of education achieved was primary (15\%), secondary (58\%) and tertiary (27\%) (Figure 7b). When compared to average data from 28 European countries, this NDI cohort has a significantly $(p=0.03)$ smaller proportion of patients achieving an academic degree (EU28: $35 \%)$. The rate of patients $\geq 25$ years of age in full employment was $73 \%$. In the age range of $25-55$ years for which comparable general population data are available, there was no significant difference for fulltime employment between this NDI cohort (78.4\%) and EU28 (80.4\%). 79\% of the patients in our cohort with an age above 30 years old were living independently (away from parental home) (Figure 7c). 


\section{Discussion}

We report on clinical, genetic and psychosocial data from patients with a diagnosis of primary NDI. To the best of our knowledge our cohort is the largest reported so far for this condition, spanning an age range of 70 years (Figure 1d) and including patients from 22 countries. More than a third of patients are adults, thereby providing robust data on final height, educational achievement, employment and living arrangements. Our results are thus of relevance to the management and prognosis of patients affected by this rare condition.

\section{Genetics}

The vast majority (86\%) of patients had genetic testing performed (Figure 1b), with causative mutations identified in almost all (96\%). This diagnostic yield is roughly similar to previously reported figures, which typically are around $90-95 \%$ [17-19]. As expected, there is a strong predominance of male patients (84\%) due to the X-linked inheritance of AVPR2 mutations. However, the proportion of patients with autosomal recessive NDI (16\%) is slightly higher than the usually reported $10 \%[1,19]$. We presume that this reflects a higher proportion of patients from consanguineous background in our cohort, as $69 \%$ of $A Q P 2$ mutations were homozygous. Of note, the prevalence of $A Q P 2$-associated NDI in patients from European centres was $12 \%$ and thus similar to previous reports.

\section{Age at diagnosis}

Most patients were diagnosed in the first year of life (Figure 2a) with the typical presentation of NDI in the first year of life with vomiting and growth failure [1]. Nevertheless, $42 \%$ of patients was diagnosed later, including $6 \%$ diagnosed as adults, the oldest one at the age of 60 years. This reflects the spectrum of severity of this disorder and there are probably several factors leading to such late diagnosis: firstly, $32 \%(\mathrm{~N}=6)$ of patients diagnosed in adult age were females with confirmed $(\mathrm{N}=3)$ or 
potential (untested or no identified mutation) AVPR2 mutations, likely reflecting skewed Xinactivation with some AVPR2 expression [20]. Next, some missense mutations may not completely abolish functionality of the encoded protein, leading to partial NDI [21]. Indeed, patients with pLoF mutations were diagnosed at younger age than those with missense variants (Figure $2 b$ ). Lastly, this may in part also reflect the health care system, as age of diagnosis was later in countries with low compared to high GDP (Figure 2d).

\section{Auxology}

Data on growth are overall reassuring: while height SDS was below the average in the paediatric age group, it was still in the normal range (Figure 3c). More importantly, final height in adults is similar to the normal population (Figure 3d), suggesting that the lower height ascertained in childhood may reflect delayed puberty, a common complication of chronic kidney disease [22]. Of interest is the significantly increased proportion of obesity in adults (Figure 3b), which was most pronounced in those with confirmed mutations. Children with NDI typically receive specialist dietetic advice to maximise caloric intake without increasing the osmotic load, so as to provide sufficient calories for normal growth, yet minimising urine output [1]. This is also reflected in the fact that $25 \%$ of children received long-term tube feeding. This treatment appears to work well during childhood as weight SDS in children was similar to the age- and sex-matched general population. However, the increased adult weight potentially reflects ongoing caloric maximisation, including perhaps from calorie-containing drinks, even when growth has finished. Our data suggest that ongoing dietetic support into adulthood may be indicated, albeit with the aim of reducing caloric input, rather than increasing it. Moreover, as obesity increases the risk for diabetes mellitus, proactive monitoring might be advisable in obese adult patients, especially since one of the cardinal symptoms of diabetes, polyuria, is already present anyway. 
Kidney function

Almost a third of patients below 20 years of age had CKD stage $\geq 2$ ( $5 \%$ stage $\geq 3$ ) and this increased to $48 \%$ ( $25 \%$ stage $\geq 3$ ) in the $>20$-year age group (Figure 4). Using the NHANESIII data for comparison in adults, the prevalence of CKD stage $\geq 2$ is significantly higher in our NDI cohort. While there are no large-scale epidemiological studies of CKD in children, data from registries suggest a prevalence of CKD around 70 per million of the age-related population $(<0.01 \%)[23,24]$. Thus, the prevalence of CKD is significantly higher in NDI patients across all age groups. This may reflect flow uropathy (as those with this complication have lower GFR), as well as kidney injury from repeated episodes of dehydration. Nevertheless, ESKD is rare and was reported in only one patient.

Drug treatment

Drugs typically used in the treatment of NDI with the aim of reducing urine output include NSAID, thiazides and amiloride [1]. A previous single centre report had suggested that these drugs may be less effective with increasing age and that many patients come off drug treatment during school age [18]. A similar decrease in drug use with age was also reported in a paediatric multicentre study [19]. Our data here appear to confirm this: while more than $80 \%$ of paediatric patients are treated with medications, this decreases to $54 \%$ in adult patients (Figure 5).

\section{Urological Complications}

Nocturnal enuresis is an important problem in paediatric NDI patients, because of the large volumes of urine produced and the attached social stigma [18]. In this study, patients achieved nocturnal continence, albeit delayed at a median age of 8 years.

Flow uropathy and bladder dysfunction, especially bladder enlargement are recognised complications of NDI, associated with the large urine volumes $[18,25]$. Our data suggest that these complications 
are present in almost half of all patients, which is similar to another multicentre cohort [19]. Importantly, the presence of this complication was associated with a lower eGFR. Yet, whether this truly reflects kidney damage from the flow uropathy, or whether patients with this complication have just more severe disease with potentially more episodes of dehydration cannot be discerned from our data.

\section{Mental Health}

Very little data on mental health problems in NDI exist. One single centre study specifically examining this issue reported that almost half of patients fulfilled criteria for a diagnosis of ADHD [2], yet in a separate cohort, a formal diagnosis of ADHD was noted in only $12.5 \%$ [18]. Our data here show a prevalence of $16 \%$, more in line with the second study, yet this may be an underestimate as patients may either not have been formally tested or the corresponding nephrologist may not have been aware of the diagnosis. In any case, this prevalence is higher than in the general population (5\%) [11]. The fact that the prevalence of ADHD is roughly similar across the genetic groups argues against a genespecific effect and instead may be related to brain injury from repeated severe dehydration or simply reflects difficulties concentrating because of constant thirst and need to go to the toilet [2].

Perhaps surprisingly, intellectual disability was the second most frequent diagnosis within the mental health disorders, reported in 9\% of patients with available data. Early reports of NDI had highlighted complications of severe mental impairment and intracranial calcifications: in one study, 3 out of 17 patients had an intelligence quotient $\geq 1$ SD below the norm and there are further reports of patients with severe mental impairment $[2,26-28]$. This is considered a complication from repeated episodes of severe dehydration that can be avoided with adequate treatment [1]. While the frequency of intellectual impairment in our report is lower than in those earlier reports, it nevertheless remains a problem. Due to the low number $(\mathrm{N}=16)$ of patients with reported intellectual disability, statistical analysis to identify potential risk factors did not provide further information. Importantly, the degree 
of intellectual disability and especially data on formal intelligence assessments were not captured in our study, so that the overall significance of this problem cannot be assessed further.

\section{Education, employment and living arrangements}

So far, virtually no data on education and employment have been reported for patients with NDI. The fact that a smaller proportion of patients achieved an academic degree compared to the European average is consistent with the report of intellectual disability in 9\%. Encouragingly, the finding that the proportion of 25-55-year old patients in full-time employment is similar to the general population and that the vast majority of $>30$-year old patients live independently from their parents argues against pervasive intellectual problems.

\section{Conclusions}

We provide clinical and social data on a large cohort of patients with NDI. Overall, these suggest a favourable long-term outcome, with patients attaining a normal final height and a similar rate of fulltime employment as the general population. While mild CKD is common, ESKD is extremely rare. However, more than half of patients suffer from urological complications and/or mental health problems, respectively. Our data inform the management and prognosis of patients with NDI.

\section{Acknowledgements}

This work was supported by The European reference network for rare kidney diseases (ERKnet).

Dr Lopez-Garcia is supported by the William Harvey Research Institute. Dr. Bockenhauer is supported by the NIHR Biomedical Research Centre at GOSH/ICH. 


\section{References}

1. Bockenhauer D, Bichet DG. Pathophysiology, diagnosis and management of nephrogenic diabetes insipidus. Nat Rev Nephrol 2015;11(10):576-588

2. Hoekstra JA, van Lieburg AF, Monnens LA, et al. Cognitive and psychosocial functioning of patients with congenital nephrogenic diabetes insipidus. Am J Med Genet 1996;61(1):81-88

3. Schwartz GJ, Munoz A, Schneider MF, et al. New equations to estimate GFR in children with CKD. J Am Soc Nephrol 2009;20(3):629-637

4. WHO Child Growth Standards based on length/height, weight and age. Acta Paediatr Suppl 2006;450:76-85

5. de Onis M, Onyango AW, Borghi E, et al. Development of a WHO growth reference for schoolaged children and adolescents. Bull World Health Organ 2007;85(9):660-667

6. Kuczmarski RJ, Ogden CL, Guo SS, et al. 2000 CDC Growth Charts for the United States: methods and development. Vital Health Stat 11 2002(246):1-190

7. Clinical guidelines on the identification, evaluation, and treatment of overweight and obesity in adults: executive summary. Expert Panel on the Identification, Evaluation, and Treatment of Overweight in Adults. Am J Clin Nutr 1998;68(4):899-917

8. Selistre L, De Souza V, Cochat P, et al. GFR Estimation in Adolescents and Young Adults. Journal of the American Society of Nephrology 2012;23(6):989-996

9. Kidney Disease: Improving Global Outcomes (KDIGO) CKD Work Group. KDIGO 2012 Clinical Practice Guideline for the Evaluation and Management of Chronic Kidney Disease. Kidney International Supplements 2013;3(1):73-90

10. Coresh J, Astor BC, Greene T, et al. Prevalence of chronic kidney disease and decreased kidney function in the adult US population: Third National Health and Nutrition Examination Survey. Am J Kidney Dis 2003;41(1):1-12

11. Polanczyk G, de Lima MS, Horta BL, et al. The worldwide prevalence of ADHD: a systematic review and metaregression analysis. Am J Psychiatry 2007;164(6):942-948

12. Eurostat. Educational attainment statistics. https://ec.europa.eu/eurostat/statisticsexplained/index.php?title=Educational attainment statistics\#level of educational attainment by age (01/05/2019; date last accessed).

13. Eurostat. Employment rate by sex and age. https://ec.europa.eu/eurostat/databrowser/view/t2020 10/default/table?lang=en $\quad$ (28/02/2020; date last accessed).

14. American Psychiatric A, American Psychiatric A, Force DSMT. Diagnostic and statistical manual of mental disorders : DSM-5. American Psychiatric Association, Placed Published: 2013.

15. 2018 WB. World Development Report 2018: Digital Dividends. Washington DC: World Bank.

16. Alonso J, Angermeyer MC, Bernert S, et al. Prevalence of mental disorders in Europe: results from the European Study of the Epidemiology of Mental Disorders (ESEMeD) project. Acta Psychiatr Scand Suppl 2004(420):21-27

17. Sasaki S, Chiga M, Kikuchi E, et al. Hereditary nephrogenic diabetes insipidus in Japanese patients: analysis of 78 families and report of 22 new mutations in AVPR2 and AQP2. Clin Exp Nephrol 2013;17(3):338-344

18. Sharma S, Ashton E, lancu D, et al. Long-term outcome in inherited nephrogenic diabetes insipidus. Clin Kidney J 2019;12(2):180-187

19. D'Alessandri-Silva C, Carpenter M, Ayoob R, et al. Diagnosis, Treatment, and Outcomes in Children With Congenital Nephrogenic Diabetes Insipidus: A Pediatric Nephrology Research Consortium Study. Front Pediatr 2019;7:550

20. Arthus MF, Lonergan M, Crumley MJ, et al. Report of 33 novel AVPR2 mutations and analysis of 117 families with X-linked nephrogenic diabetes insipidus. J Am Soc Nephrol 2000;11(6):1044-1054 
21. Bockenhauer $\mathrm{D}$, Carpentier $\mathrm{E}$, Rochdi $\mathrm{D}$, et al. Vasopressin type 2 receptor V88M mutation: molecular basis of partial and complete nephrogenic diabetes insipidus. Nephron Physiol 2010;114(1):p1-10

22. Haffner D, Zivicnjak M. Pubertal development in children with chronic kidney disease. Pediatr Nephrol 2017;32(6):949-964

23. Ardissino G, Daccò V, Testa S, et al. Epidemiology of Chronic Renal Failure in Children: Data From the ItalKid Project. Pediatrics 2003;111(4):e382-e387

24. Areses Trapote R, Sanahuja Ibanez MJ, Navarro M, et al. [Epidemiology of chronic kidney disease in Spanish pediatric population. REPIR II Project]. Nefrologia 2010;30(5):508-517

25. Shalev H, Romanovsky I, Knoers NV, et al. Bladder function impairment in aquaporin-2 defective nephrogenic diabetes insipidus. Nephrol Dial Transplant 2004;19(3):608-613

26. van Lieburg AF, Knoers NV, Monnens LA. Clinical presentation and follow-up of 30 patients with congenital nephrogenic diabetes insipidus. J Am Soc Nephrol 1999;10(9):1958-1964

27. Hillman DA, Neyzi O, Porter P, et al. Renal (vasopressin-resistant) diabetes insipidus; definition of the effects of a homeostatic limitation in capacity to conserve water on the physical, intellectual and emotional development of a child. Pediatrics 1958;21(3):430-435

28. Vest M, Talbotnb, Crawford JD. Hypocaloric dwarfism and hydronephrosis in diabetes insipidus. Am J Dis Child 1963;105:175-181 
Table 1. Auxology.

Reported weight and height SDS for paediatric and adult patients according to genetic groups. Significant differences $\left({ }^{*},{ }^{* *} ; \mathrm{p}<0.05\right)$ were observed when the Negative group was compared to both $A V P R 2$ and $A Q P 2$.

\begin{tabular}{|l|c|c|}
\hline Genetic Group (N) & Weight SDS & Height SDS \\
\hline $\begin{array}{l}\text { Negative } \\
\quad \text { Paeds (7) }\end{array}$ & $-0.26(-0.77-1.01)$ & $0.50(-0.75-1.73)^{* *}$ \\
$\quad$ Adults (5) & $0.06(-0.51-0.89)^{*}$ & $0.12(-0.83-0.47)$ \\
\hline Untested & & \\
$\quad$ Paeds (25) & $-0.1(-1.98-1.39)$ & $-0.68(-1.98-0.30)$ \\
$\quad$ Adults (18) & $1.0(0.05-1.52)$ & $-0.37(-0.93-0.16)$ \\
\hline AVPR2 & $-0.11(-1.24-0.68)$ & $-0.90(-1.56 \text { to }-0.19)^{* *}$ \\
$\quad$ Paeds (148) & $1.36(0.49-1.79)^{*}$ & $-0.30(-1.07-0.30)$ \\
$\quad$ Adults (67) & & \\
\hline AQP2 & $-0.29(-1.83-0.96)$ & $-1.31(-1.83 \text { to }-0.27)^{* *}$ \\
$\quad$ Paeds (25) & $1.32(0.8-1.89)^{*}$ & $-0.48(-1.41-0.54)$ \\
\hline Adults (17)
\end{tabular}




\section{Figure legends}

\section{Figure 1. Demographic aspects of our cohort}

Shown are selected characteristics of our cohort ( $\mathrm{N}=315$, unless otherwise stated): A) Gender, B) Genetic group (for details see text) and $C$ ) reported ethnicity $(N=300)$. D) Boxplot graph detailing age at last follow-up according to genetic group (Negative $(N=12)$, Untested $(N=45), A V P R 2(N=216)$ and AQP2 (N=42).

\section{Figure 2. Age at diagnosis}

Shown is the age at diagnosis in respect to several selected characteristics: A) genetic group, note that $90 \%$ of patients were diagnosed before the age of 10 years. B) mutation type. Patients with predicted loss of function variants (pLoF) were diagnosed significantly earlier than those with missense variants. C) gender and genetic group. Males ( $N=199)$ with AVPR2 pathogenic variants were diagnosed earlier than females (N=12). D) Gross domestic product per capita (GDP). Patients living in countries with higher GDP ( $N=186$; GDP >15.000\$/year) were diagnosed earlier compared with those in lower GDP countries ( $N=121, \mathrm{GDP}<15.000 \$ /$ year $)$.

\section{Figure 3. Auxology}

Shown are data on height, weight and body mass index (BMI) according to selected parameters: A) Boxplot representing weight SDS in paediatric patients according to their genetic group: Negative $(\mathrm{N}=7)$, Untested $(\mathrm{N}=25)$, AVPR2 $(\mathrm{N}=148)$ and AQP2 $(\mathrm{N}=25)$. Note the roughly similar distribution across the genetic groups. B) BMI of adult patients (Underweight <18.5, Normal 18.5-24.9, Overweight 25.029.9 and Obese $\geq 30.0 \mathrm{~kg} / \mathrm{m}^{2}$ ). Note the high prevalence of overweight and obese patients in this NDI cohort. C) Height SDS in children according to genetic group. Note that $15 \%$ of children had a height $<2.0 \mathrm{SDS})$ and that both median (-0.9) and IQR (-1.7 to -0.1) are lower than expected for age. Moreover, height SDS was significantly lower in those with confirmed mutations (AVPR2, N=147 and AQP2, $N=24$ ) compared to those without identified mutation (Negative, $N=7$ and Untested, $N=25$ ). D) Adult (final) height according to genetic group. Note the essentially normal final height across all genetic groups: Negative ( $N=4)$, Untested $(N=18)$, AVPR2 ( $N=63)$ AQP2 ( $N=14)$.

\section{Figure 4. Kidney Function}

Shown are data for eGFR and corresponding CKD changes. B) CKD stage distribution in paediatric (220 years, $N=199$ ) and $B$ ) adult patients ( $N=87)$. Note that $5 \%$ of children and $25 \%$ of adults are in CKD stage $\geq 3$. C) eGFR in adult patients ( $>20$ years, $N=87$ ) against age at last follow up. Note the kidney function decline estimated at $1.4 \mathrm{~mL} / \mathrm{min} / 1.73 \mathrm{~m}^{2}$ per year with a starting eGFR of $110 \mathrm{~mL} / \mathrm{min} / 1.73 \mathrm{~m}^{2}$ at age 20 years. D) CKD stages in adult patients compared to a reference population (NHANES 3). Note the significantly $(p<0.001)$ higher prevalence of CKD in the NDI cohort in both age groups: $20-40$ years $(\mathrm{N}=57)$ and $40-60$ years $(\mathrm{N}=19)$. E) eGFR of paediatric and $\mathrm{F}$ ) adult patients according to their countries' per capita GDP. Note that eGFR was significantly lower in children living in countries with low $(<15.000 \$, \mathrm{~N}=77)$ compared to high GDP $(>15.000 \$, \mathrm{~N}=122)$. This difference was no longer noted in adult patients. 


\section{Figure 5. Treatment}

Shown is the distribution of treatment with specific medications according to age. A) Histograms with distribution of treatment with thiazide diuretics and B) non-steroidal anti-inflammatory drugs (NSAID) according to age. Note that both treatments are predominantly prescribed during the paediatric age. $73 \%$ of patients receiving a thiazide $(\mathrm{N}=217)$ were below the age of 18 years of age compared to $91 \%$ of NSAIDs ( $\mathrm{N}=98$ ) for same age group. $\mathrm{C}$ ) Number of medications prescribed according to three age groups: infants (under 2 years, $\mathrm{N}=17$ ), paediatrics ( $2-18$ years, $\mathrm{N}=200$ ) and adults ( $>18$ years, $\mathrm{N}=98$ ). Note that almost half (44\%) of adult patients received no medications, compared to $15 \%$ of children and that the majority of paediatric patients were treated with combination of medications.

\section{Figure 6. Urological Complications}

Shown is the frequency of selected associated urological complications. A) Frequency of manifestation of flow uropathy (hydronephrosis and/or bladder dysfunction). Note that $45 \%$ of patients were reported to have evidence of flow uropathy. B) Flow uropathy and eGFR. Patients with flow uropathy had a lower mean eGFR compared to those without (90 versus $103 \mathrm{~mL} / \mathrm{min} / 1.73 \mathrm{~m}^{2}$ ). C) Histogram showing the distribution of age (years) at which nocturnal continence was achieved $(\mathrm{N}=85)$. Note that the median (IQR) age was 8 (6-12) years with a wide range of 3-21 years.

\section{Figure 7. Psychosocial aspects}

Shown are data on mental health, education and living arrangements. A) Mental health problems reported in the cohort $(\mathrm{N}=178)$. Note that more than a third $(36 \%)$ of patients were reported to have mental health problems, with ADHD being the most frequent single diagnosis. B) Highest completed level of education in patients from EU-28 countries aged 25-54 years ( $N=41)$ compared to EU-28 reference data. Note that NDI patients did not achieve as many academic degrees as expected from the reference population. C) Employment and living arrangements in adult patients. Note that $73 \%$ of patients older than 25 years $(\mathrm{N}=62)$ were in full-time employment and that $79 \%$ of those older than 30 years $(\mathrm{N}=52$ ) were living independent from their parents. No significant differences were observed regarding employment status and living arrangements between our patients and average EU-28 population. 